\title{
Identification of risk factors for sepsis-associated mortality by gene expression profiling analysis
}

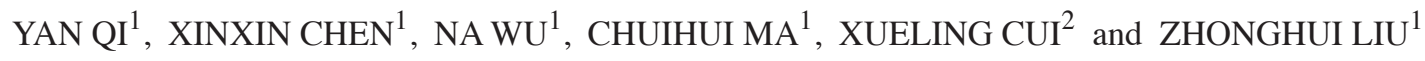 \\ Departments of ${ }^{1}$ Immunology and ${ }^{2}$ Genetics, College of Basic Medical Sciences, Jilin University, \\ Changchun, Jilin 130021, P.R. China
}

Received July 19, 2017; Accepted December 1, 2017

DOI: $10.3892 / \mathrm{mmr} .2018 .8491$

\begin{abstract}
Sepsis is a common cause of mortality due to systemic infection. Although numerous studies have investigated this life-threatening condition, there remains a lack of suitable markers to evaluate the severity of sepsis. The present study focused on the identification of risk factors for sepsis-associated mortality by genome-wide expression profiling. Initially, the GEO2R web tool was used to identify the differentially expressed genes (DEGs) between sepsis survivors and nonsurvivors. It was identified that the upregulated DEGs in the nonsurvivors compared with survivors were highly enriched in the type I interferon (IFN-I) signaling pathway. Furthermore, the associations of the upregulated genes were analyzed by STRING and the results demonstrated that a set of proteins in IFN-I signaling pathway closely interacted with each other. To further investigate whether the IFN-I signaling pathway is dysregulated in a subset of patients with a high risk of mortality due to sepsis, in this case neonates, the DEGs between the cord blood mononuclear cells of neonates and adult peripheral blood mononuclear cells were analyzed. It was identified that DEGs were not enriched in IFN-I signaling in the blood of untreated neonates and adults; however, IFN-I signaling was upregulated in the lipopolysaccharide (LPS)-treated cord blood mononuclear cells of healthy neonates compared with the LPS-treated peripheral blood mononuclear cells of adults. In addition, these data revealed that the proteins involved in the IFN-I signaling pathway possessed a higher number of interacting partners. These
\end{abstract}

Correspondence to: Professor Zhonghui Liu, Department of Immunology, College of Basic Medical Sciences, Jilin University, 126 Xinmin Street, Changchun, Jilin 130021, P.R. China

E-mail: liuzh@jlu.edu.cn

Professor Xueling Cui, Department of Genetics, College of Basic Medical Sciences, Jilin University, 126 Xinmin Street, Changchun, Jilin 130021, P.R. China

E-mail: cxl@jlu.edu.cn

Key words: sepsis, gene expression profiling, risk factor, type I interferon signaling results indicated that upregulated IFN-I signaling may be a high-risk factor for mortality due to sepsis.

\section{Introduction}

Sepsis is a systemic inflammatory response syndrome that occurs when a bacterial, viral or fungal infection spreads to the bloodstream and induces life-threatening organ dysfunction (1). Without immediate and aggressive treatment, sepsis can rapidly cause tissue damage, organ failure, and even death. More than 5 million people die from sepsis every year worldwide (2). Despite numerous advances in fundamental and clinical research, the mortality rate for sepsis remains high (3-4). Over the last 30 years, over 100 clinical trials have failed to indicate a survival benefit for patients with severe sepsis, and the failure is due, at least partially, to host heterogeneity (5-8). Patients vary in their circumstances, and a more precise assessment of sepsis is required.

Risk factors associated with deterioration or mortality may be used to diagnose and evaluate the severity of sepsis. Thereby, the subset of patients with a high risk of mortality for sepsis may receive additional, aggressive therapies. Thus far, a number of biomarkers have been widely used for the diagnosis, prognosis and treatment of sepsis (5,10-12). For example, $\mathrm{C}$-reactive protein (CRP) is an acute phase protein, which increases rapidly in response to most forms of inflammation, infection, and tissue damage. High levels of CRP are associated with the risk of sepsis, cardiovascular disease and stroke (12-14). While CRP is broadly used for clinical diagnosis of acute sepsis, it lacks the capacity to differentiate between infective and non-infective inflammation and have low specificity for severe sepsis $(15,16)$. Severe sepsis is often attributed to immune dysregulation, and the imbalance between pro- and anti-inflammatory cytokines may serve a crucial role in the pathogenesis of sepsis (17). Patients who express high levels of interleukin (IL)-6 have an increased risk of mortality $(18,19)$. Although the combination of IL-6 and CRP plasma biomarkers may be helpful in sepsis diagnosis, recent studies demonstrated that IL- 6 and CRP are not ideal as they lack sufficient sensitivity and specificity (20-22).

Genome expression profiling is a potential approach to discover novel risk factors based on microarray technology and bioinformatics (23). Microarray technology enables researchers to gain insights into signaling pathways and gene 
networks that may participate in sepsis development (24-26). The present study focused on the identification of risk factors for predicting sepsis deterioration by genome-wide expression profiling. Differentially expressed genes (DEGs) between sepsis survivors and nonsurvivors were analyzed, and type I interferon (IFN-I) signaling was identified as an important risk factor for sepsis-associated mortality.

\section{Materials and methods}

Gene expression profiles. Two gene expression profiles (GSE54514 and GSE3140) were downloaded from public functional genomics data repository Gene Expression Omnibus (GEO; www.ncbi.nlm.nih.gov/geo/) (27-28). The array data from GSE54514 were performed on the platform of Illumina Human HT-12 v3.0 Expression BeadChip (GPL6947; Illumina, Inc., San Diego, CA, USA). This dataset contained 53 blood samples, including 26 samples from sepsis survivors, 9 samples from sepsis nonsurvivors and 18 samples from healthy controls.

The gene expression profile of GSE3140 was performed on the platform of an Affymetrix GeneChip Human HG-Focus Target Array (GPL201; Affymetrix; Thermo Fisher Scientific, Inc., Waltham, MA, USA). Prior to the mRNA expression profiling, blood samples were collected from 6 healthy adult volunteers and 6 healthy, full-term infants, and RNA was isolated from cord blood and adult peripheral blood mononuclear cells of blood samples following incubation with or without lipopolysaccharide (LPS).

Screening of DEGs. DEGs were identified using GEO2R (http://www.ncbi.nlm.nih.gov/geo/geo2r/). GEO2R is an R-based web tool for performing differential gene expression analysis in the GEO data repository (29). The adjusted P-values (adj. P) were adjusted using the Benjamini and Hochberg false discovery rate method (30). Genes with adj. $\mathrm{P}<0.05$ and $\mid \log \mathrm{FCl}>1$ were considered to be DEGs, and the heatmap of DEGs was generated using the heatmap visualization tool Morpheus (software.broadinstitute.org/morpheus/).

Functional enrichment of DEGs. The Gene Ontology (GO) defines classes used to describe gene function and associations between biology concepts (31). It classifies functions according to three aspects: Molecular Function, Cellular Component and Biological Process. In the present study, GO enrichment analysis of DEGs was performed using Gene Ontology Consortium (www.geneontology.org/), with $\mathrm{P}<0.05$ indicating a significantly enriched term (32).

Protein-protein interaction (PPI) network. STRING is a database and web resource of experimental and predicted PPIs. STRING provides a score for each interaction, and these scores are indicators of confidence and rank from $0-1$. The online STRING 10.5 database (string-db.org/) was used to analyze protein interactions and a confidence score $>0.4$ was used as the cut-off criterion (33).

\section{Results}

Identification of DEGs between sepsis survivors and nonsurvivors. To determine the early risk factors for sepsis-associated mortality, the GSE54514 dataset was downloaded from the GEO database and GEO2R was used to identify the DEGs between blood samples from sepsis survivors and nonsurvivors. A heatmap of DEGs was subsequently generated by Morpheus. The results demonstrated that a total of 18 DEGs were identified, including 14 upregulated genes and 4 downregulated genes in nonsurvivors compared with survivors (Fig. 1).

IFN-I signaling is upregulated in nonsurvivors of sepsis. The identified DEGs were functionally enriched by GO analysis using the GOC website with $\mathrm{P}<0.05$ as the threshold. As demonstrated in Table I, DEGs that were upregulated in the nonsurvivors group compared with the survivors group were highly enriched in 15 pathways, including 'type I interferon signaling pathway', 'cellular response to type I interferon', 'response to type I interferon', 'negative regulation of viral genome replication' and 'regulation of viral genome replication'. To further investigate the association between the upregulated genes, STRING was used to construct the PPI network. As demonstrated in Fig. 2, a set of proteins from IFN-I signaling pathway closely interacted with each other, which indicates that they may be implicated in sepsis biology due to their interactions. These results indicated that upregulated IFN-I signaling may be a risk factor for sepsis-associated mortality.

IFN-I signaling is upregulated in blood samples from $L P S$-treated healthy neonates. IFN-I signaling is crucial for the host defense; however, its role in sepsis remains controversial. The results of the present study indicated that, during early sepsis, upregulated IFN-I signaling may be a marker for an increased risk of mortality. Therefore, the present study also investigated whether this signaling pathway was dysregulated in a subset of patients with a high risk of sepsis-induced mortality (34). It is widely accepted that neonates suffer higher sepsis mortality rates compared with adults (35), therefore, DEGs between the cord blood mononuclear cells of healthy neonates and adult peripheral blood mononuclear cells were analyzed and numerous DEGs were identified by GEO2R. However, instead of IFN-1 signaling, DEGs upregulated in healthy neonates compared with healthy adults were largely enriched in 'protoporphyrinogen IX metabolic process', 'gas transport', 'oxygen transport', 'erythrocyte development' and 'porphyrin-containing compound biosynthetic process', among others (Table II).

Furthermore, upregulated DEGs between the LPS-treated cord blood mononuclear cells of healthy neonates and LPS-treated peripheral blood mononuclear cells of healthy adults were identified and analyzed by GO enrichment. Consistent with the hypothesis that the IFN-I pathway may be upregulated in a subset of patients with sepsis that normally exhibit a higher risk of mortality, 'type I interferon signaling pathway' and 'response to interferon- $\alpha$ ' were upregulated in the LPS-treated blood mononuclear cells of healthy neonates compared with adults (Table III). Subsequently, the associations among the upregulated genes were analyzed by STRING; 183 proteins were demonstrated to be involved in the interaction network and were separated into several clusters, and the proteins involved in IFN-I signaling appeared to exhibit a 
Table I. Enriched pathways of differentially expressed genes that were upregulated in the whole blood of sepsis nonsurvivors compared with survivors.

\begin{tabular}{llccc}
\hline Pathway ID & \multicolumn{1}{c}{ Pathway description } & Count & Fold enrichment & P-value \\
\hline GO:0060337 & Type I interferon signaling pathway & 7 & $>100$ & $1.14 \times 10^{-9}$ \\
GO:0071357 & Cellular response to type I interferon & 7 & $>100$ & $1.14 \times 10^{-9}$ \\
GO:0034340 & Response to type I interferon & 7 & $>100$ & $1.72 \times 10^{-9}$ \\
GO:0045071 & Negative regulation of viral genome replication & 4 & 84.91 & $1.21 \times 10^{-3}$ \\
GO:0045069 & Regulation of viral genome replication & 4 & 53.84 & $7.33 \times 10^{-3}$ \\
GO:1903901 & Negative regulation of viral life cycle & 4 & 49.61 & $1.01 \times 10^{-2}$ \\
GO:0048525 & Negative regulation of viral process & 4 & 39.42 & $2.51 \times 10^{-2}$ \\
GO:0051607 & Defense response to virus & 6 & 38.96 & $5.96 \times 10^{-5}$ \\
GO:0043901 & Negative regulation of multi-organism process & 5 & 34.07 & $2.48 \times 10^{-3}$ \\
GO:0009615 & Response to virus & 7 & 30.54 & $1.40 \times 10^{-5}$ \\
GO:0043903 & Regulation of symbiosis, encompassing mutualism & 6 & 22.53 & $1.49 \times 10^{-3}$ \\
& through parasitism & 5 & & $3.05 \times 10^{-2}$ \\
GO:0050792 & Regulation of viral process & 7 & 6.37 & $5.84 \times 10^{-4}$ \\
GO:0098542 & Defense response to other organism & 6 & 17.68 & $7.61 \times 10^{-3}$ \\
GO:0043900 & Regulation of multi-organism process & 7 & 16.13 & $1.09 \times 10^{-3}$ \\
GO:0019221 & Cytokine-mediated signaling pathway & 7.03 & \\
\hline
\end{tabular}

Count refers to the number of enriched genes in each Biological Process GO term. The top 15 Biological Process terms were selected based on the fold enrichment score. GO, gene ontology.

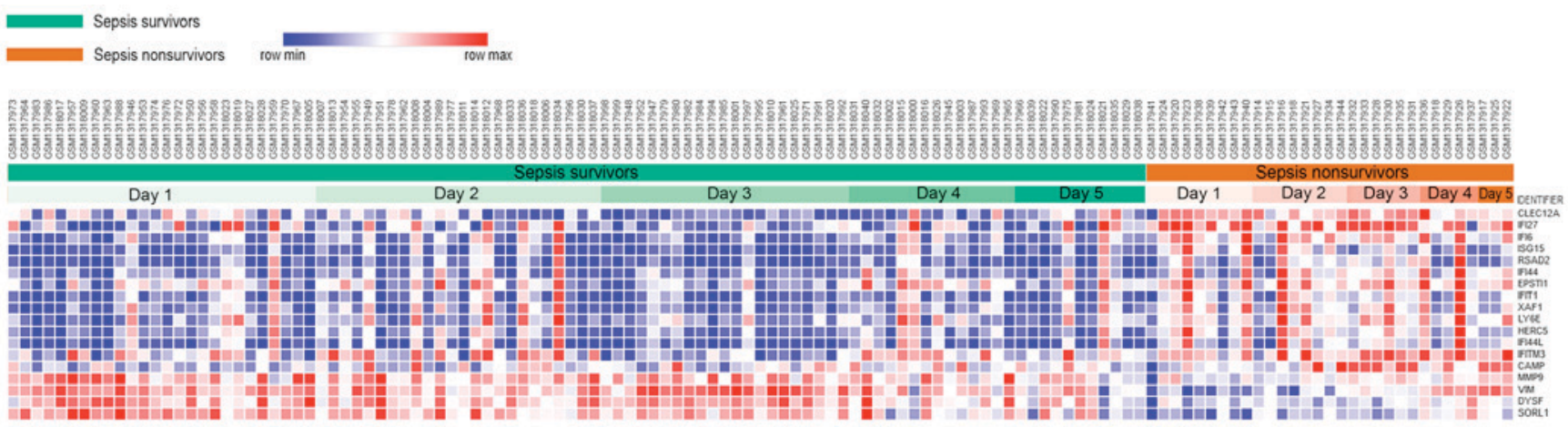

Figure 1. Cluster analysis of differentially expressed genes in the GSE54514 dataset. Group names and sample names are on the horizontal axis, while gene symbols are on right vertical axis. Red color represents upregulated genes and blue color represents downregulated genes.

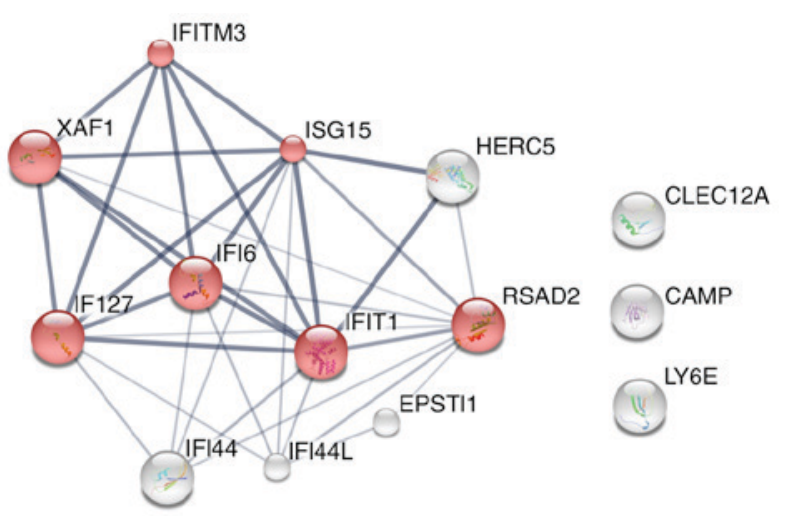

Figure 2. Protein-protein interaction network. The association among differentially expressed genes that were upregulated in the sepsis nonsurvivors group compared with survivors was analyzed by STRING. Line thickness indicates the strength of data support, proteins are presented as nodes and the proteins that are involved in type I interferon signaling are presented in red. higher number of interactions (Fig. 3). These results indicated that IFN-I signaling may be upregulated in a subset of patients with sepsis, in this case neonates, that have a higher risk of mortality.

\section{Discussion}

Sepsis is an important cause of mortality from infection, and although numerous studies have been performed concerning this life-threatening condition, there remains a lack of effective treatment and an assessment of risk factors to assist in the diagnostic process. The present study focused on the identification of potential risk factors for sepsis-associated mortality by genome-wide expression profiling. The results demonstrated that DEGs that were upregulated in sepsis nonsurvivors compared with survivors were highly enriched in the IFN-I signaling pathway. The associations between the 
Table II. Enriched pathways of differentially expressed genes that were upregulated in the untreated cord blood mononuclear cells of healthy neonates compared with the untreated peripheral blood mononuclear cells of healthy adults.

\begin{tabular}{|c|c|c|c|c|}
\hline Pathway ID & Pathway description & Count & Fold enrichment & P-value \\
\hline GO:0046501 & Protoporphyrinogen IX metabolic process & 11 & 33.45 & $3.23 \times 10^{-4}$ \\
\hline GO:0015669 & Gas transport & 19 & 25.82 & $1.26 \times 10^{-5}$ \\
\hline GO:0015671 & Oxygen transport & 14 & 21.9 & $3.53 \times 10^{-2}$ \\
\hline GO:0048821 & Erythrocyte development & 24 & 17.89 & $1.59 \times 10^{-3}$ \\
\hline GO:0006779 & Porphyrin-containing compound biosynthetic process & 26 & 14.15 & $4.58 \times 10^{-2}$ \\
\hline GO:0006778 & Porphyrin-containing compound metabolic process & 38 & 12.91 & $2.46 \times 10^{-3}$ \\
\hline GO:0061515 & Myeloid cell development & 44 & 12.54 & $5.65 \times 10^{-4}$ \\
\hline GO:0046686 & Response to cadmium ion & 56 & 9.86 & $4.18 \times 10^{-3}$ \\
\hline GO:0033013 & Tetrapyrrole metabolic process & 59 & 9.35 & $6.40 \times 10^{-3}$ \\
\hline GO:0034614 & Cellular response to reactive oxygen species & 119 & 8.24 & $2.01 \times 10^{-6}$ \\
\hline GO:0000302 & Response to reactive oxygen species & 198 & 7.12 & $4.36 \times 10^{-9}$ \\
\hline GO:0042542 & Response to hydrogen peroxide & 107 & 6.88 & $2.46 \times 10^{-3}$ \\
\hline GO:0034599 & Cellular response to oxidative stress & 203 & 6.34 & $3.76 \times 10^{-7}$ \\
\hline GO:0009636 & Response to toxic substance & 210 & 5.84 & $4.50 \times 10^{-6}$ \\
\hline GO:0030099 & Myeloid cell differentiation & 190 & 5.49 & $2.21 \times 10^{-4}$ \\
\hline
\end{tabular}

Count refers to the number of enriched genes in each Biological Process GO term. The top 15 Biological Process terms were selected based on the fold enrichment score. GO, gene ontology.

upregulated genes were analyzed by STRING and the results demonstrated that the proteins were also highly associated with IFN-I signaling pathway. Therefore, it was hypothesized that a dysregulated IFN-I signaling pathway may be associated with a high risk of sepsis-associated mortality.

IFN-Is, which include IFN- $\alpha$ and IFN- $\beta$, trigger IFN-I signaling by binding to the IFN- $\alpha / \beta$ receptor (IFNAR), stimulating the Janus kinase/signal transducer and activator of transcription pathway and initiating the transcription of IFN-stimulated genes, which mediate various anticellular effects by modulating cell viability and function (36). The IFN-I signaling pathway is well known for its protective roles in the majority of viral infections, while its functions in bacterial infection remain controversial. This controversy arises as certain studies have reported that IFN-I signaling served a critical role in host protection against bacterial infection and that the development of bacteremia during sepsis was enhanced in the mice that lack IFNAR $(37,38)$, while other studies indicated that IFNAR-deficient mice were partially protected against lethality in multiple inflammatory models, including endotoxemia-induced shock, cecal ligation and puncture-induced sepsis, and colon ascendens stent peritonitis-induced sepsis (39-41). In addition, IFN-I signaling may exert toxic effects during sepsis by negatively regulating neutrophil recruitment and suppressing adaptive immunity, leading to inefficient control of infections and eventual mortality $(40,42,43)$.

Although these findings highlight the critical role of the IFN-I signaling pathway in sepsis, there remains a lack of reliable evidence from clinical studies. The results of the present study demonstrated that upregulated IFN-I signaling may be a high-risk factor for sepsis-associated mortality; however, it remains to be elucidated whether dysregulated
IFN-I signaling may affect the mortality of patients with sepsis. Although a potential risk factor for short-term mortality in sepsis is provided, the survivors still suffer a high risk of long-term mortality for months or years. Although IFN-I signaling was upregulated in several survivors compared with the others, no information concerning their long-term mortality is available due to a lack of data, therefore a more comprehensive scrutiny of clinical research concerning the effect of the IFN-I signaling pathway in sepsis is required.

As the initial results of the present study indicated that upregulated IFN-I signaling may be a potential marker for a higher risk of sepsis-associated mortality, it was further investigated whether this signaling pathway was dysregulated in a subset of patients that possess a higher risk of sepsis-associated mortality. It is established that neonates suffer a higher rate of sepsis-associated mortality compared with adults; therefore, DEGs between the untreated cord blood mononuclear cells of healthy neonates and untreated peripheral blood mononuclear cells of adults were analyzed (44). There were numerous DEGs between neonates and adults; however, the DEGs were not enriched in the IFN-1 signaling pathway. Subsequently, upregulated DEGs between LPS-treated cord blood mononuclear cells of healthy neonates and LPS-treated peripheral blood mononuclear cells of adults were identified and, consistent with the hypothesis, 'type I interferon signaling pathway' and 'response to interferon- $\alpha$ ' were upregulated in the LPS-treated cells of healthy neonates compared with adults. Furthermore, the associations among the upregulated genes were analyzed and the results demonstrated that the proteins associated with the IFN-I signaling possessed a higher number of interactions and may function together in pathological processes. 
Table III. Enriched pathways of differentially expressed genes that were upregulated in the LPS-treated cord blood mononuclear cells of healthy neonates compared with LPS-treated peripheral blood mononuclear cells of healthy adults.

\begin{tabular}{|c|c|c|c|c|}
\hline Pathway ID & Pathway description & Count & Fold enrichment & P-value \\
\hline GO:0045086 & Positive regulation of interleukin-2 biosynthetic process & 12 & 15.13 & $3.20 \times 10^{-2}$ \\
\hline GO:0045589 & Regulation of regulatory $\mathrm{T}$ cell differentiation & 19 & 12.74 & $2.81 \times 10^{-3}$ \\
\hline GO:0035455 & Response to interferon- $\alpha$ & 20 & 12.11 & $4.12 \times 10^{-3}$ \\
\hline GO:0045076 & Regulation of interleukin-2 biosynthetic process & 18 & 11.77 & $2.57 \times 10^{-2}$ \\
\hline GO:0045624 & Positive regulation of T-helper cell differentiation & 19 & 11.15 & $3.65 \times 10^{-2}$ \\
\hline GO:0043372 & Positive regulation of CD4-positive, $\alpha-\beta$ T cell differentiation & 25 & 10.89 & $1.91 \times 10^{-3}$ \\
\hline GO:0002719 & $\begin{array}{l}\text { Negative regulation of cytokine production involved in } \\
\text { immune response }\end{array}$ & 23 & 10.53 & $1.16 \times 10^{-2}$ \\
\hline GO:2000516 & Positive regulation of CD4-positive, $\alpha-\beta$ T cell activation & 29 & 10.44 & $6.07 \times 10^{-4}$ \\
\hline GO:0002828 & Regulation of type 2 immune response & 27 & 10.09 & $3.60 \times 10^{-3}$ \\
\hline GO:0032743 & Positive regulation of interleukin- 2 production & 31 & 9.76 & $1.12 \times 10^{-3}$ \\
\hline GO:0042346 & Positive regulation of NF- $\mathrm{KB}$ import into nucleus & 25 & 9.68 & $2.13 \times 10^{-2}$ \\
\hline GO:0019835 & Cytolysis & 25 & 9.68 & $2.13 \times 10^{-2}$ \\
\hline GO:0032731 & Positive regulation of interleukin- $1 \beta$ production & 30 & 9.08 & $8.53 \times 10^{-3}$ \\
\hline GO:0032663 & Regulation of interleukin-2 production & 51 & 8.9 & $2.99 \times 10^{-6}$ \\
\hline GO:0043370 & Regulation of CD4-positive, $\alpha-\beta$ T cell differentiation & 38 & 8.76 & $7.76 \times 10^{-4}$ \\
\hline GO:2000514 & Regulation of CD4-positive, $\alpha-\beta$ T cell activation & 44 & 8.25 & $3.85 \times 10^{-4}$ \\
\hline GO:0045070 & Positive regulation of viral genome replication & 33 & 8.25 & $1.84 \times 10^{-2}$ \\
\hline GO:0045071 & Negative regulation of viral genome replication & 52 & 8.15 & $3.48 \times 10^{-5}$ \\
\hline GO:0046635 & Positive regulation of $\alpha-\beta$ T cell activation & 53 & 7.99 & $4.40 \times 10^{-5}$ \\
\hline GO:0042108 & Positive regulation of cytokine biosynthetic process & 61 & 7.94 & $3.97 \times 10^{-6}$ \\
\hline GO:0048247 & Lymphocyte chemotaxis & 42 & 7.93 & $2.07 \times 10^{-3}$ \\
\hline GO:0045069 & Regulation of viral genome replication & 82 & 7.75 & $1.23 \times 10^{-8}$ \\
\hline GO:0002704 & Negative regulation of leukocyte mediated immunity & 47 & 7.73 & $7.78 \times 10^{-4}$ \\
\hline GO:0060337 & Type I interferon signaling pathway & 65 & 7.45 & $9.73 \times 10^{-6}$ \\
\hline GO:0071357 & Cellular response to type I interferon & 65 & 7.45 & $9.73 \times 10^{-6}$ \\
\hline
\end{tabular}

Count refers to the number of enriched genes in each Biological Process GO term. The top 25 Biological Process terms were selected based on the fold enrichment score. GO, gene ontology.

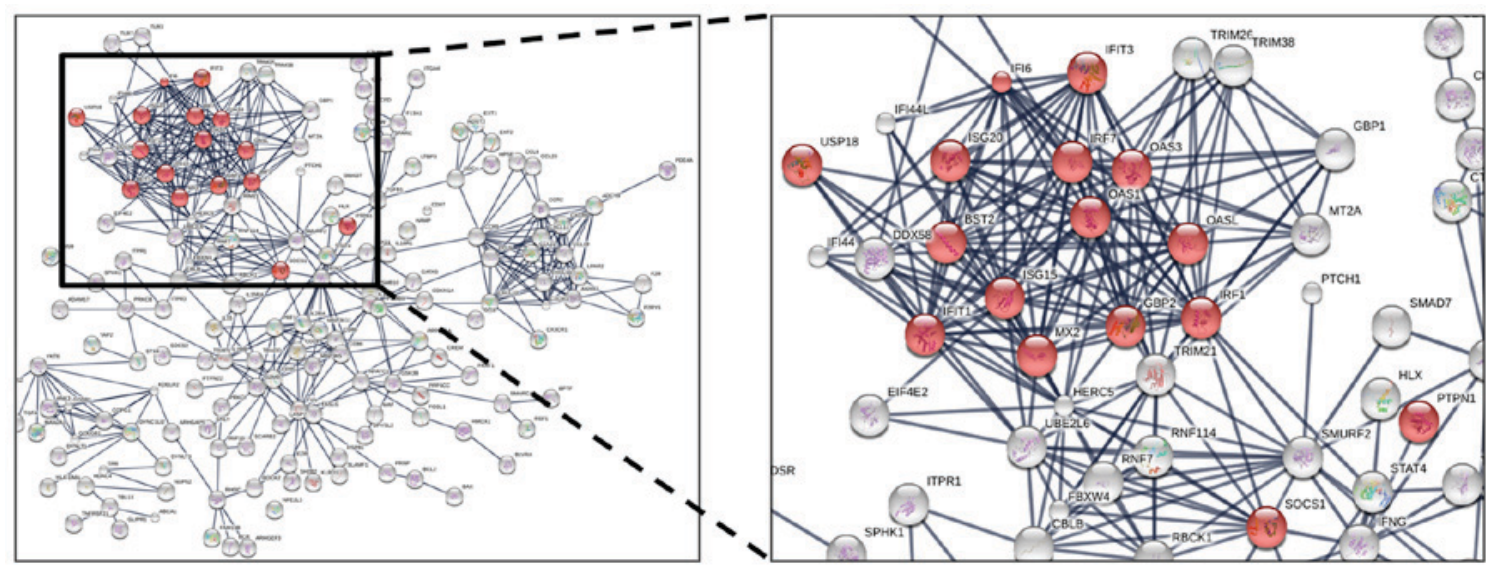

Figure 3. Protein-protein interaction network. The association among differentially expressed genes that were upregulated in LPS-treated cord blood mononuclear cells from healthy neonates compared with LPS-treated peripheral blood mononuclear cells from healthy adults was analyzed by STRING. Line thickness indicates the strength of data support, proteins are presented as nodes and the proteins that are involved in type I interferon signaling are presented in red. LPS, lipopolysaccharide.

In conclusion, the present findings suggest that upregulated IFN-I signaling pathway may be a risk factor for sepsis-associated mortality, but further studies are needed to confirm the current results. 


\section{Acknowledgements}

The present study was supported by the National Natural Science Foundation of China (grant nos. 31500738 and 81273199), the Health and Family Planning Commission of Jilin Province (grant no. 2014Z067) and the Union Projects of Jilin University and Xinjiang Medical University (for Liu Z).

\section{References}

1. Singer CS, Deutschman CS, Seymour CW, Shankar-Hari M, Annane D, Bauer M, Bellomo R, Bernard GR, Chiche JD, Coopersmith CM, et al: The third international consensus definitions for sepsis and septic shock (Sepsis-3). JAMA 315: 801-810, 2016.

2. Rudd KE, Delaney A and Finfer S: Counting sepsis, an imprecise but improving science. JAMA 318: 1228-1229, 2017.

3. Rhodes A, Evans LE, Alhazzani W, Levy MM, Antonelli M, Ferrer R, Kumar A, Sevransky JE, Sprung CL, Nunnally ME, et al: Surviving sepsis campaign: International guidelines for management of sepsis and septic shock: 2016. Crit Care Med 45: 486-552, 2017.

4. Shankar-Hari M, Phillips GS, Levy ML, Seymour CW, Liu VX, Deutschman CS, Angus DC, Rubenfeld GD and Singer M; Sepsis Definitions Task Force: Developing a new definition and assessing new clinical criteria for septic shock: For the third international consensus definitions for sepsis and septic shock (Sepsis-3). JAMA 315: 775-787, 2016.

5. van der Poll T, van de Veerdonk FL, Scicluna BP and Netea MG: The immunopathology of sepsis and potential therapeutic targets. Nat Rev Immunol 17: 407-420, 2017.

6. Seeley EJ and Bernard GR: Therapeutic targets in sepsis: Past, present, and future. Clin Chest Med 37: 181-189, 2016.

7. Hotchkiss RS, Monneret G and Payen D: Immunosuppression in sepsis: A novel understanding of the disorder and a new therapeutic approach. Lancet Infect Dis 13: 260-268, 2013.

8. Dellinger RP: Severe sepsis trials: Why have they failed? Minerva Anestesiol 65: 340-345, 1999.

9. Gotts JE and Matthay MA: Sepsis: Pathophysiology and clinical management. BMJ 353: i1585, 2016

10. Seymour CW and Rosengart MR: Septic shock: Advances in diagnosis and treatment. JAMA 314: 708-717, 2015.

11. McLean AS, Tang B and Huang SJ: Investigating sepsis with biomarkers. BMJ 350: h254, 2015.

12. Vashist SK, Venkatesh AG, Marion Schneider E, Beaudoin C, Luppa PB and Luong JH: Bioanalytical advances in assays for C-reactive protein. Biotechnol Adv 34: 272-290, 2016.

13. Ridker PM: A test in context: High-sensitivity c-reactive protein. J Am Coll Cardiol 67: 712-723, 2016.

14. Póvoa P, Coelho L, Almeida E, Fernandes A, Mealha R, Moreira P and Sabino H: Pilot study evaluating C-reactive protein levels in the assessment of response to treatment of severe bloodstream infection. Clin Infect Dis 40: 1855-1857, 2005.

15. Fraunberger P, Wang Y, Holler E, Parhofer KG, Nagel D, Walli AK and Seidel D: Prognostic value of interleukin 6 , procalcitonin, and C-reactive protein levels in intensive care unit patients during first increase of fever. Shock 26: 10-12, 2006.

16. Schneider CP, Yilmaz Y, Kleespies A, Jauch KW and Hartl WH: Accuracy of procalcitonin for outcome prediction in unselected postoperative critically ill patients. Shock 31: 568-573, 2009

17. Chousterman BG, Swirski FK and Weber GF: Cytokine storm and sepsis disease pathogenesis. Semin Immunopathol 39: 517-528, 2017.

18. Mikacenic C, Price BL, Harju-Baker S, O'Mahony DS, Robinson-Cohen C, Radella F, Hahn WO, Katz R, Christiani DC, Himmelfarb J, et al: A Two-biomarker model predicts mortality in the critically III with Sepsis. Am J Respir Crit Care Med 196: 1004-1011, 2017.

19. Qi Y, Ge J, Ma C, Wu N, Cui X and Liu Z: Activin A regulates activation of mouse neutrophils by Smad3 signalling. Open Biol 7: 160342, 2017.

20. Ettinger M, Calliess T, Kielstein JT, Sibai J, Brückner T, Lichtinghagen $\mathrm{R}$, Windhagen $\mathrm{H}$ and Lukasz A: Circulating biomarkers for discrimination between aseptic joint failure, low-grade infection, and high-grade septic failure. Clin Infect Dis 61: 332-341, 2015.
21. Buttaro MA, Tanoira I, Comba F and Piccaluga F: Combining C-reactive protein and interleukin-6 may be useful to detect periprosthetic hip infection. Clin Orthop Relat Res 468: 3263-3267, 2010.

22. Elgeidi A, Elganainy AE, Abou Elkhier N and Rakha S: Interleukin-6 and other inflammatory markers in diagnosis of periprosthetic joint infection. Int Orthop 38: 2591-2595, 2014.

23. Rung J and Brazma A: Reuse of public genome-wide gene expression data. Nat Rev Genet 14: 89-99, 2013.

24. Maslove DM and Wong HR: Gene expression profiling in sepsis: Timing, tissue, and translational considerations. Trends Mol Med 20: 204-213, 2014.

25. Wong HR: Clinical review: Sepsis and septic shock-the potential of gene arrays. Crit Care 16: 204, 2012.

26. Tang BM, Huang SJ and McLean AS: Genome-wide transcription profiling of human sepsis: A systematic review. Crit Care 14: R237, 2010

27. Parnell GP, Tang BM, Nalos M, Armstrong NJ, Huang SJ, Booth DR and McLean AS: Identifying key regulatory genes in the whole blood of septic patients to monitor underlying immune dysfunctions. Shock 40: 166-174, 2013.

28. Koch L, Linderkamp O, Ittrich C, Benner A and Poeschl J: Gene expression profiles of adult peripheral and cord blood mononuclear cells altered by lipopolysaccharide: Neonatology 93: 87-100, 2008.

29. Barrett T, Wilhite SE, Ledoux P, Evangelista C, Kim IF, Tomashevsky M, Marshall KA, Phillippy KH, Sherman PM, Holko M, et al: NCBI GEO: Archive for functional genomics data sets-update. Nucleic Acids Res 41 (Database Issue): D991-D995, 2013.

30. Benjamini Y, Drai D, Elmer G, Kafkafi N and Golani I: Controlling the false discovery rate in behavior genetics research. Behav Brain Res 125: 279-284, 2001.

31. Rhee SY, Wood V, Dolinski K and Draghici S: Use and misuse of the gene ontology annotations. Nat Rev Genet 9: 509-515, 2008.

32. Munoz-Torres M and Carbon S: Get GO! Retrieving GO data using AmiGO, QuickGO, API, Files, and tools. Methods Mol Biol 1446: 149-160, 2017.

33. Szklarczyk D, Morris JH, Cook H, Kuhn M, Wyder S, Simonovic M, Santos A, Doncheva NT, Roth A, Bork P, et al: The STRING database in 2017: Quality-controlled protein-protein association networks, made broadly accessible. Nucleic Acids Res 45 (D1): D362-D368, 2017

34. Trinchieri G: Type I interferon: Friend or foe? J Exp Med 207: 2053-2063, 2010

35. Kan B, Razzaghian HR and Lavoie PM: An immunological perspective on neonatal sepsis. Trends Mol Med 22: 290-302, 2016.

36. Schreiber $\mathrm{G}$ and Piehler J: The molecular basis for functional plasticity in type I interferon signaling. Trends Immunol 36: $139-149,2015$.

37. LeMessurier KS, Häcker H, Chi L, Tuomanen E and Redecke V: Type I interferon protects against pneumococcal invasive disease by inhibiting bacterial transmigration across the lung. PLoS Pathog 9: e1003727, 2013.

38. Mancuso G, Midiri A, Biondo C, Beninati C, Zummo S, Galbo R, Tomasello F, Gambuzza M, Macrì G, Ruggeri A, et al: Type I IFN signaling is crucial for host resistance against different species of pathogenic bacteria. J Immunol 178: 3126-3133, 2007.

39. Mahieu T, Park JM, Revets H, Pasche B, Lengeling A, Staelens J, Wullaert A, Vanlaere I, Hochepied T, van Roy F, et al: The wild-derived inbred mouse strain SPRET/Ei is resistant to LPS and defective in IFN-beta production. Proc Natl Acad Sci USA 103: 2292-2297, 2006.

40. Dejager L, Vandevyver S, Ballegeer M, Van Wonterghem E, An LL, Riggs J, Kolbeck R and Libert C: Pharmacological inhibition of type I interferon signaling protects mice against lethal sepsis. J Infect Dis 209: 960-970, 2014.

41. Weighardt H, Kaiser-Moore S, Schlautkötter S, Rossmann-Bloeck T, Schleicher U, Bogdan C and Holzmann B: Type I IFN modulates host defense and late hyperinflammation in septic peritonitis. J Immunol 177: 5623-5630, 2006.

42. Schwandt T, Schumak B, Gielen GH, Jüngerkes F, Schmidbauer P, Klocke K, Staratschek-Jox A, van Rooijen N, Kraal G, Ludwig-Portugall I, et al: Expression of type I interferon by splenic macrophages suppresses adaptive immunity during sepsis. EMBO J 31: 201-213, 2012.

43. McNab F, Mayer-Barber K, Sher A, Wack A and O'Garra A: Type I interferons in infectious disease. Nat Rev Immunol 15: 87-103, 2015.

44. Ginsburg AS, Meulen AS and Klugman KP: Prevention of neonatal pneumonia and sepsis via maternal immunisation. Lancet Glob Health 2: e679-e680, 2014. 